\title{
Direct Liquid to Crystal Transition in a Quasi-Two-Dimensional Colloidal Membrane
}

\author{
Thomas Gibaud ${ }^{\dagger}$ and Doru Constantin $*, \ddagger$ \\ †Univ. Lyon, ENS de Lyon, Univ. Claude Bernard, CNRS, Laboratoire de Physique, \\ F-69342 Lyon, France \\ $\ddagger$ Laboratoire de Physique des Solides, CNRS, Univ. Paris-Sud, Université Paris-Saclay, \\ 91405 Orsay Cedex, France
}

E-mail: doru.constantin@u-psud.fr

\begin{abstract}
Using synchrotron-based small angle x-ray scattering, we study rigid $f d$ viruses assembled into isolated monolayers from mixtures with a non-absorbing polymer, which acts as osmotic agent. As the polymer concentration increases we observe a direct liquid to crystal transition, without an intermediate hexatic phase, in contrast with many other similar systems, such as concentrated DNA phases or packings of surfactant micelles. We tentatively attribute this effect to the difference in stiffness. The liquid phase can be well described by a hard-disk fluid, while we model the crystalline one as a hexagonal harmonic lattice and we evaluate its elastic constants.
\end{abstract}



How does the ordering transition occur in two-dimensional systems of objects with isotropic interaction? Over the last halfcentury, this deceivingly simple question received complex answers that reveal considerable hidden subtlety. In particular, according to the celebrated Kosterlitz-Thouless-HalperinNelson-Young (KTHNY) scenario, ${ }^{1}$ crystallization is preceded by the formation of an intermediate hexatic phase, characterized by two order parameters: a short-range positional one and a (sixfold) quasi-long-range orientational one.

Experimental measurements of the interaction between particles and of their degree of order have been performed on a wide variety of systems, such as adsorbed noble gases, ${ }^{2-4}$ monolayers of colloidal particles, ${ }^{5-8}$ protein layers $^{9}$ or nano-objects inserted within lipid or surfactant bilayers (e.g. peptides ${ }^{10}$ or inorganic nanoparticles. ${ }^{11,12}$ ) In many of these "flat" systems (with a transverse size comparable to the typical in-plane distance), the hexatic phase is indeed observed. ${ }^{2-8}$

A more complex case is that of packed elongated particles, where the out-of-plane degrees of freedom can also be important. The hexatic phase is present, for instance, in stacks of colloidal platelets, ${ }^{13}$ in systems of self-assembled micelles, ${ }^{14}$ but also in dense phases of doublestranded (ds) DNA, ${ }^{15-17}$ which are similar to the very compact state of DNA encoutered, for instance, in bacteriophage capsids. ${ }^{18}$ 
Here, we use $f d$ viruses, quite similar to dsDNA: they are monodisperse, chiral, charged and rigid rodlike objects that assemble under the influence of depleting agents into a wide variety of self-assembled structures, such as tactoids, ${ }^{19}$ colloidal membranes, ${ }^{20,21}$ twisted ribbons $^{22}$ and membrane rafts. ${ }^{23}$ We concentrate here on colloidal membranes, i.e. monolayers where the rods are parallel to the layer normal.

Despite its rich morphology, this system has only been studied at the macroscopic scale and in the fluid regime, via optical microscopy observation. Structural characterization, over length scales comparable to the distance between rods, is rendered difficult by the small size and reduced scattering power of the assemblies, although it has been carried out on bulk phases of related systems. ${ }^{24,25}$

We perform small-angle X-ray scattering studies (SAXS) at a high-brilliance synchrotron beamline on suspensions of such objects. The number concentration $n_{2 \mathrm{D}}$ of rods in the plane of the membrane is controlled by the bulk polymer concentration. As $n_{2 \mathrm{D}}$ increases, we observe a first-order transition between an isotropic phase and a crystal phase. There is no hexatic phase, in contrast with many other twodimensional systems, such as dsDNA, and with the bulk phase of the semi-flexible wild-type $f d$ viruses. ${ }^{25}$

As semiflexible rodlike particles we used the monodisperse filamentous bacteriophage $f d$ Y21M with a contour length of $880 \mathrm{~nm}$, a diameter of $6.6 \mathrm{~nm}$ and a persistence length of $9.9 \pm 1.6 \mu m .{ }^{26} f d$-Y21M viruses were synthesized using standard biological protocols ${ }^{27}$ and dispersed at a concentration $c_{V}$ ranging from 0.5 to $10 \mathrm{mg} / \mathrm{mL}$ in $20 \mathrm{mM}$ TRIS buffer at pH 8.0 and $100 \mathrm{mM} \mathrm{NaCl}$.

In aqueous suspension, these virus particles exhibit purely repulsive interactions and behave as model system with respect to the Onsager theory. ${ }^{26}$ We mixed the viruses with a non absorbing polymer, Dextran (molecular weight $500 \mathrm{kDa}$, Sigma Aldrich) at a final virus concentration $c_{V}=5 \mathrm{mg} / \mathrm{mL}$ and polymer concentrations in a range $c_{D}=0-58.5 \mathrm{mg} / \mathrm{mL}$. Adding polymers to a dilute isotropic suspension of $f d$ $\mathrm{Y} 21 \mathrm{M}$ induces attractive interactions between the rods via the depletion mechanism. ${ }^{28}$

The membrane properties are mainly controled by the depletant concentration, ${ }^{20,22}$ and their diameter ranges from 1 to 50 microns. ${ }^{20}$ The line tension at the edge of the membranes is always too high to self-assemble monodispersed membranes with a well-defined diameter. ${ }^{22}$ The thermal fluctuations of the membranes provide an entropic repulsion that keeps them isolated from each other along their normal. However, at high depletant concentrations, the induced attraction may be strong enough to overcome the entropic repulsion and the membranes tend to stack on top of each other ${ }^{20}$ and eventually crystalize.

We prepared bulk samples of concentrated membranes in round glass capillaries, with a nominal outer diameter of $1 \mathrm{~mm}$ and 10 micronthick walls, purchased from WJM-Glas (Berlin, Germany). The capillaries were filled with the sample solution and sealed at the top to prevent evaporation. Some of them were mildly centrifuged for 5 minutes at $2000 \mathrm{~g}$ to concentrate the membranes at the bottom of the capillary and thus increase the strength of the scattered signal. The sediment occupies at least $1 / 5$ of the capillary length, so the local concentration does not exceed $c_{V}=25 \mathrm{mg} / \mathrm{mL}$. We checked that there is no significant difference between the signal of the initial solutions and that of the centrifuged ones (see the Supplemental Material $^{29}$ for more details.)

SAXS measurements were performed on the SWING beamline of the SOLEIL synchrotron (Saint-Aubin, France.) The sample-to-detector distance was $2 \mathrm{~m}$ and the wavelength $\lambda=1 \AA$, covering a scattering vector range $0.005<q<$ $0.5 \AA^{-1}$. The measurements were performed at $22{ }^{\circ} \mathrm{C}$. The capillaries were placed vertically. For the centrifuged ones, we made several measurements at different heights in the sedimented phase, at its upper interface and in the supernatant.

The intensity measured in the supernatant was used as a reference signal for that measured in the sediment.

The corrected intensity was then divided by the (orientationally averaged) form factor of the virus measured in isotropic solutions ${ }^{29}$ to yield 
the in-plane averaged structure factor $S(q)$. The structure factors are shown in Figure 1 for Dextran concentrations $c_{D}$ between 29.5 and $58.5 \mathrm{mg} / \mathrm{mL}$. At lower $c_{D}$ we do not detect the formation of membranes (the solutions remain clear) and the scattering signal is weak and relatively flat.

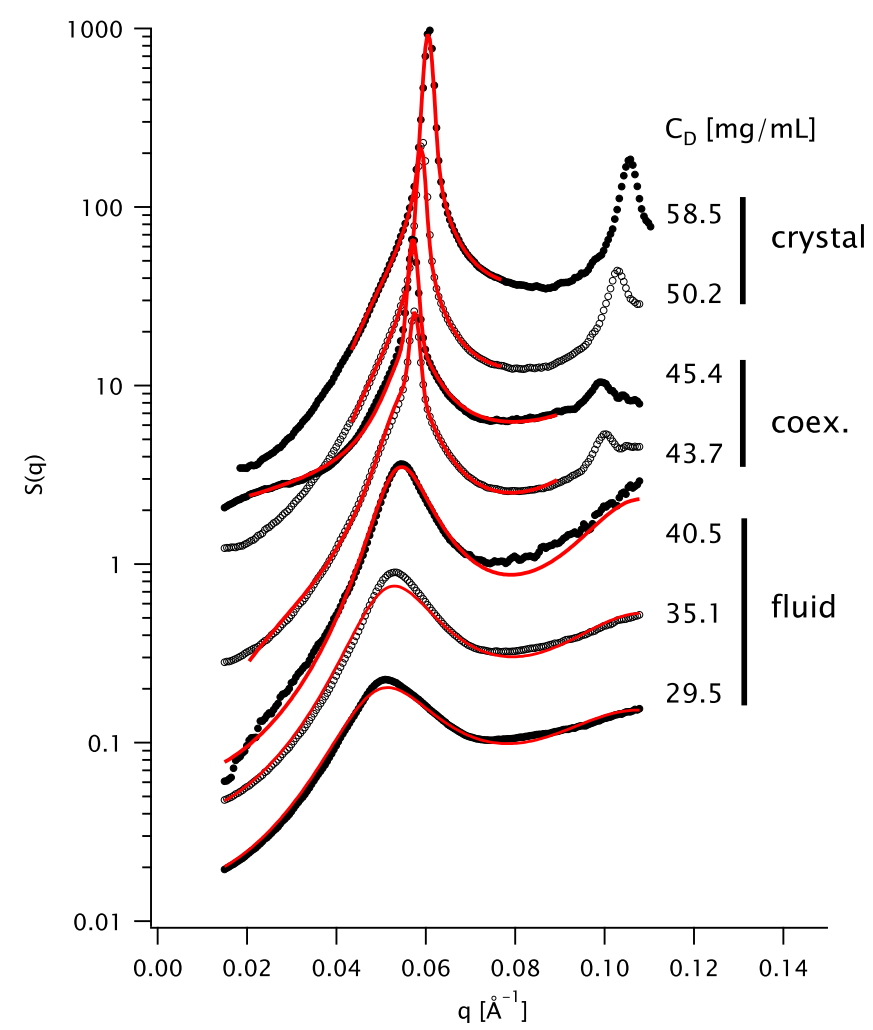

Figure 1: Structure factors $S(q)$ obtained by SAXS (open dots) for several Dextran concentrations, indicated alongside the curves, as well as the phase assignment. The fits with the appropriate models are shown as solid lines (see the text for details.) The curves are vertically shifted for clarity.

A two-dimensional system of isotropic particles can exhibit three phases: fluid, crystalline and hexatic. For the fluid phase we used the structure factor of hard disks, ${ }^{31}$ which has a simple analytical expression and yields two parameters: the hard disk radius $R_{\mathrm{HD}}$ and the in-plane number concentration $n_{2 \mathrm{D}}$ (see Figure 2). From the low- $q$ limit $S(q \rightarrow 0)$ of the fitted structure factor we extract the (twodimensional) osmotic pressure $\Pi_{2 \mathrm{D}}$.

We describe the structure factor of the crystalline phase by the harmonic model of
Dutta and Sinha ${ }^{33}$ (denoted in the following as D \& S), averaged over the azimuthal angle (in the plane of the membrane), since the recorded intensity is integrated over all orientations. There is no need to account for the experimental resolution, since this parameter would be correlated to the domain size (see the original paper for details.)

In the hexatic phase, many authors described the peaks by a Lorentzian function for simplicity: this choice yields an analytical functional form for the azimuthally averaged structure factor given by Heiney et al. ${ }^{34}(\mathrm{H})$, which is close to a square-root Lorentzian function. ${ }^{4}$ With respect to the original model, we convolute the peak function with a Gaussian to account for the experimental resolution. For completeness, we also included in the analysis the Pearson VII function $(\mathrm{P})$ used by Grelet to model the signal of the hexatic phase in bulk systems. ${ }^{25}$

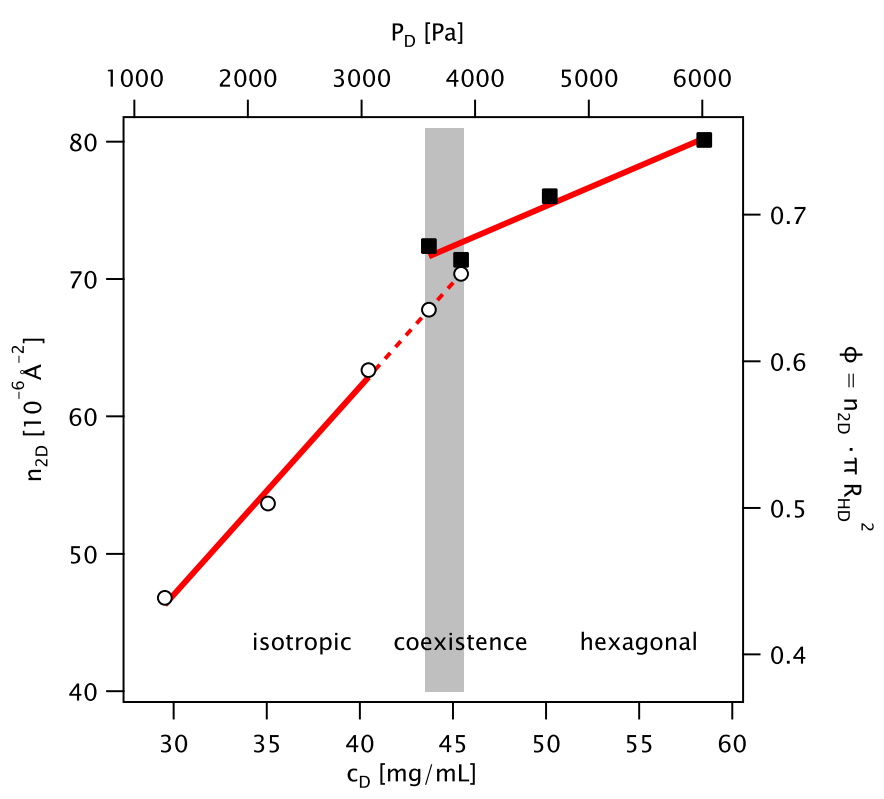

Figure 2: In-plane number density of rods within the membrane $n_{2 \mathrm{D}}$ as a function of the Dextran concentration $c_{D}$. The osmotic pressure $P_{D}$ imposed by the polymer ${ }^{35}$ is shown as top axis. In the isotropic phase $(\bigcirc), n_{2 \mathrm{D}}$ is a free parameter of the hard-disk model, while in the hexagonal one $(\boldsymbol{\square})$ it is computed from the peak position. At coexistence $\left(c_{D}=43.7\right.$ and $45.4 \mathrm{mg} / \mathrm{mL}$ ), the lower concentration is linearly extrapolated from the isotropic values, while the higher is a free fit parameter. ${ }^{29}$ 
For $c_{D}=43.7 \mathrm{mg} / \mathrm{mL}$ and above, we fitted the curves in the range 0.043-0.077 $\AA^{-1}$ (around the first peak $q_{10}$ ) using the three models described above: $\mathrm{D} \& \mathrm{~S}, \mathrm{H}$, and $\mathrm{P}$ respectively (see Figure 3 ). In all cases, we add to the models a linear slope and a constant contribution: $\alpha\left(q-q_{10}\right)+\beta$, to account for imperfect background subtraction and for other sources of signal within the sample. Each model has six fit parameters, including $\alpha$ and $\beta$.

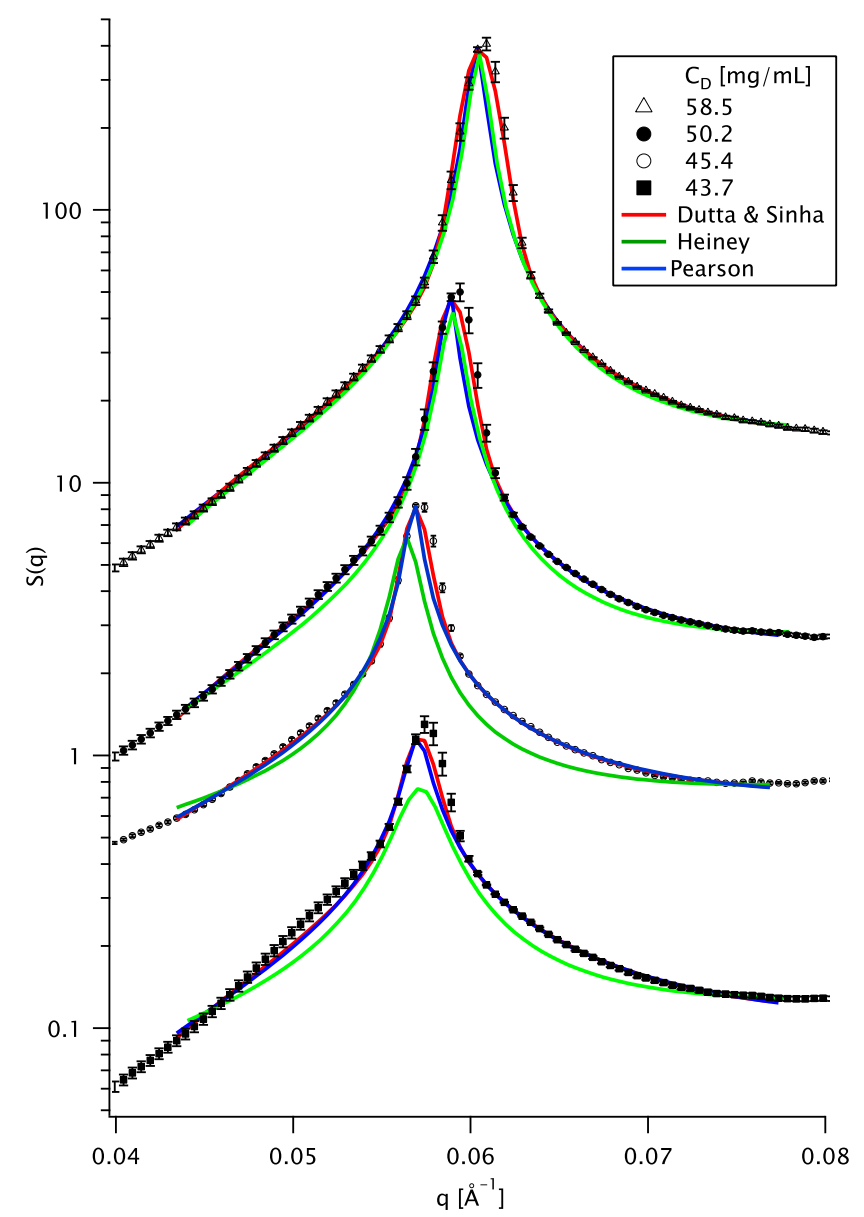

Figure 3: The top four curves in Fig. 1 (symbols) and fits with the three models (solid lines, different colors.)

In Table 1 we present the goodness-of-fit function $\chi^{2}$ (divided by the number of data points $N_{\text {pts }}$ in the fitting range) for each curve and with each model. This parameter quantifies the agreement between data and model: we expect $\chi^{2} / N_{\text {pts }} \sim 1$ for a good fit, $\chi^{2} / N_{\text {pts }}>1$ signals an imperfect fit (the model is inaccurate or the data exhibits systematic uncertainties), while $\chi^{2} / N_{\text {pts }} \ll 1$ generally results from overestimat- ing the uncertainty of the data. To ascertain the importance of the linear term added to the models we also perform the fits where we set $\alpha=0$ (columns labeled "no slope".) The huge improvement in $\chi^{2} / N_{\text {pts }}$ justifies our use of this term. Clearly, the harmonic crystal model of Dutta and Sinha provides the best description of the four curves. However, while this agreement is excellent for the two higher concentrations, as shown by $\chi^{2} / N_{\text {pts }}<1$, it is clearly worse for $c_{D}=43.7$ and $45.4 \mathrm{mg} / \mathrm{mL}$, where $\chi^{2} / N_{\text {pts }}=1.75$ and 4.59 , respectively, because the model underestimates the data in the range 0.047-0.055 $\AA^{-1}$. This also holds true for models $\mathrm{H}$ and $\mathrm{P}$.

Since none of the single-phase models fit the structure factor for $c_{D}=43.7$ and $45.4[\mathrm{mg} / \mathrm{mL}]$, we tried to describe it as coexistence between the hexagonal phase found at higher concentrations and the hard-disk liquid phase found at lower concentrations. We therefore use the sum of the Dutta \& Sinha model and the liquid model. The latter contribution has three parameters: the hard-disk radius $R_{\mathrm{HD}}$ and the in-plane number density $n_{2 \mathrm{D}}$ are obtained by extrapolation from the fits at lower concentrations, while the overall amplitude is free to vary. We therefore have a single additional coefficient with respect to the six in the D \& S model. The best fit is shown as red line in Fig. 4 (and also in Fig. 1). For comparison, we plot as black line the best D \& S fit.

The agreement is markedly better for the coexistence system: at $c_{D}=43.7 \mathrm{mg} / \mathrm{mL}$, adding one fit parameter decreases the $\chi^{2} / N_{\text {pts }}$ from 1.75 to 1.08 , and over a much wider range. For comparison, trying to fit over the same range with only the $\mathrm{D} \& \mathrm{~S}$ model yields $\chi^{2} / N_{\mathrm{pts}}=$ 27.0, while the hard-disk liquid model yields $\chi^{2} / N_{\text {pts }}=33.3$ with fixed $R_{\mathrm{HD}}$ and $n_{2 \mathrm{D}}$ and $\chi^{2} / N_{\text {pts }}=15.6$ if these two parameters are allowed to vary (in which case they do not move more than $4 \%$ away from the initially fixed values.) Similar improvement in $\chi^{2} / N_{\text {pts }}$ (from 4.59 to 2.44 ) is obtained for $c_{D}=45.4 \mathrm{mg} / \mathrm{mL}$. We conclude that the best description of these two data points is given by the coexistence model. 
Table 1: Goodness-of-fit function $\chi^{2}$ (divided by the number of data points $N_{\mathrm{pts}}$ in the fitting range) for the three models described in the text: Dutta and Sinha (DS), Heiney et al. (H) and Pearson $(\mathrm{P})$, with or without a linear slope term.

\begin{tabular}{|c||c|c|c|c|c|c|}
\hline \multirow{2}{*}{$c_{D}[\mathrm{mg} / \mathrm{mL}]$} & \multicolumn{5}{|c|}{$\chi^{2} / N_{\text {pts }}$} \\
\cline { 2 - 7 } & $\mathbf{D ~ \& ~ S ~}$ & $\mathrm{H}$ & $\mathrm{P}$ & $\mathrm{D} \&$ S (no slope) & $\mathrm{H}$ (no slope) & $\mathrm{P}$ (no slope) \\
\hline 58.5 & $\mathbf{0 . 7 0}$ & 2.82 & 3.15 & 133.8 & 168.9 & 105.8 \\
\hline 50.2 & $\mathbf{0 . 9 3}$ & 3.62 & 2.04 & 72.3 & 68.8 & 57.7 \\
\hline 45.4 & $\mathbf{4 . 5 9}$ & 22.63 & 8.14 & 132.3 & 78.1 & 98.1 \\
\hline 43.7 & $\mathbf{1 . 7 5}$ & 4.92 & 2.30 & 18.1 & 13.0 & 12.8 \\
\hline
\end{tabular}
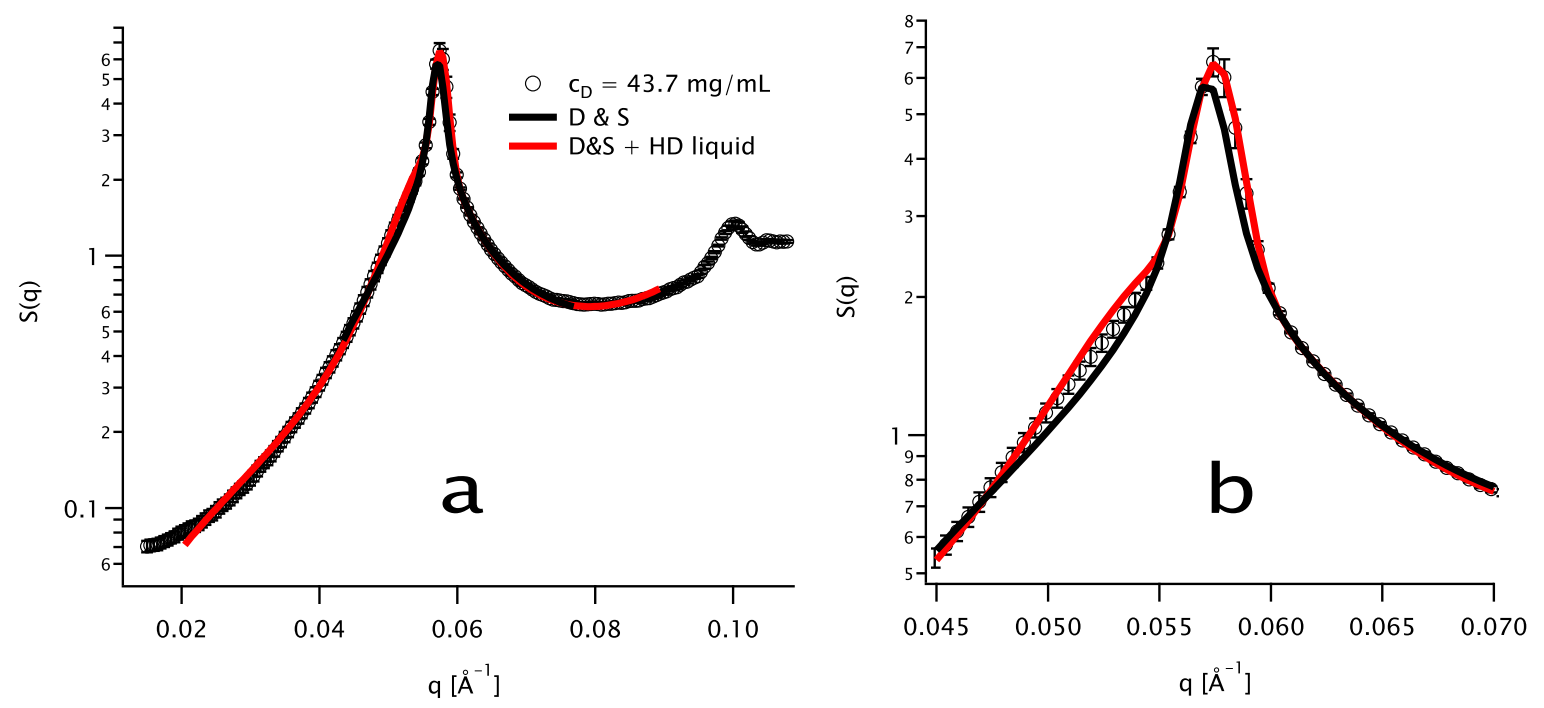

Figure 4: Structure factor at $c_{D}=43.7 \mathrm{mg} / \mathrm{mL}$ (open dots) and fits with the Dutta \& Sinha model (black solid line) and the coexistence model (red solid line). a - full range. b - detail of the peak region.

The best values for the relevant fit parameters and their associated uncertainties (at the $68.3 \%$ confidence level) are shown in Table 2. $L_{\mathrm{DS}}$ is the domain size in the Dutta and Sinha model (see the Supporting Information for the definitions.) The $n_{2 \mathrm{D}}$ is common to the two models, being obtained from the peak position $q_{10}$ in the hexagonal phase as $n_{2 \mathrm{D}}=\frac{\sqrt{3}}{8 \pi^{2}} q_{10}^{2}$. At coexistence, $n_{2 \mathrm{D}}$ of the isotropic contribution is fixed as discussed in the text and only the value corresponding to the hexagonal phase is listed. The hard-disk radius $R_{\mathrm{HD}}$ at coexistence is also fixed.

A question we cannot answer at this time is whether the coexistence is a genuine feature of the phase diagram (in which case it would imply that the transition is first-order) or an artifact due to sample heating by the x-ray beam and the system is in fact fully crystalline. We cannot rule out the latter possibility, although we limited the beam effect by reducing the exposure time for these two samples to the minimum achievable time (5 ms.)

An important parameter of the crystalline phase is the exponent $\eta(q, T)$, which describes the power law divergence of the diffuse scattering around the Bragg peaks. It is given by the elastic moduli of the phase: ${ }^{36}$

$$
\eta(q, T)=\frac{k_{B} T q^{2}}{4 \pi \mu} \frac{3 \mu+\lambda}{2 \mu+\lambda}=\frac{k_{B} T q^{2}}{8 \pi \mu}(3-\sigma)
$$

where $\mu$ (the shear modulus) and $\lambda$ are the Lamé coefficients and $\sigma$ is Poisson's ratio in two 
Table 2: Best values and associated uncertainties for the relevant fit parameters: Dutta and Sinha with linear slope term in the hexagonal phase, hard-disk liquid in the isotropic phase and their sum in the coexistence range.

\begin{tabular}{|c||c|c|c|c|}
\hline \multirow{2}{*}{$c_{D}[\mathrm{mg} / \mathrm{mL}]$} & \multicolumn{4}{|c|}{ Value \pm Uncertainty } \\
\cline { 2 - 5 } & $n_{2 \mathrm{D}}\left[10^{-6} \AA^{-1}\right]$ & $\eta$ & $L_{\mathrm{DS}}\left[10^{3} \AA\right]$ & $R_{\mathrm{HD}}[\AA]$ \\
\hline 58.5 & $80.12 \pm 0.05$ & $0.282 \pm 0.005$ & $2.9 \pm 0.1$ & \\
\hline 50.2 & $76.04 \pm 0.06$ & $0.39 \pm 0.01$ & $3.4 \pm 0.2$ & \\
\hline 45.4 & $71.40 \pm 0.07$ & $0.11 \pm 0.01$ & $2.9 \pm 0.1$ & 54.15 \\
\hline 43.7 & $72.40 \pm 0.07$ & $0.16 \pm 0.01$ & $2.8 \pm 0.1$ & 54.97 \\
\hline 40.5 & $63.37 \pm 0.03$ & & & $55.08 \pm 0.01$ \\
\hline 35.1 & $53.66 \pm 0.06$ & & & $54.15 \pm 0.01$ \\
\hline 29.5 & $46.80 \pm 0.07$ & & & $54.64 \pm 0.01$ \\
\hline
\end{tabular}

dimensions. ${ }^{1}$ In the following, we will denote by

$$
\eta=\eta\left(q_{10}, T\right)=\frac{4 \pi}{3} \frac{k_{B} T}{\mu d^{2}} \frac{3 \mu+\lambda}{2 \mu+\lambda}
$$

the value of this parameter at room temperature and at the position of the first Bragg peak of the hexagonal lattice, $q_{10}=\frac{4 \pi}{\sqrt{3} d}$, where $d \simeq$ $12 \mathrm{~nm}$ is the distance between first neighbors in the lattice. Close to $q_{10}, S(q) \sim 1 /\left|q-q_{10}\right|^{2-\eta}$.

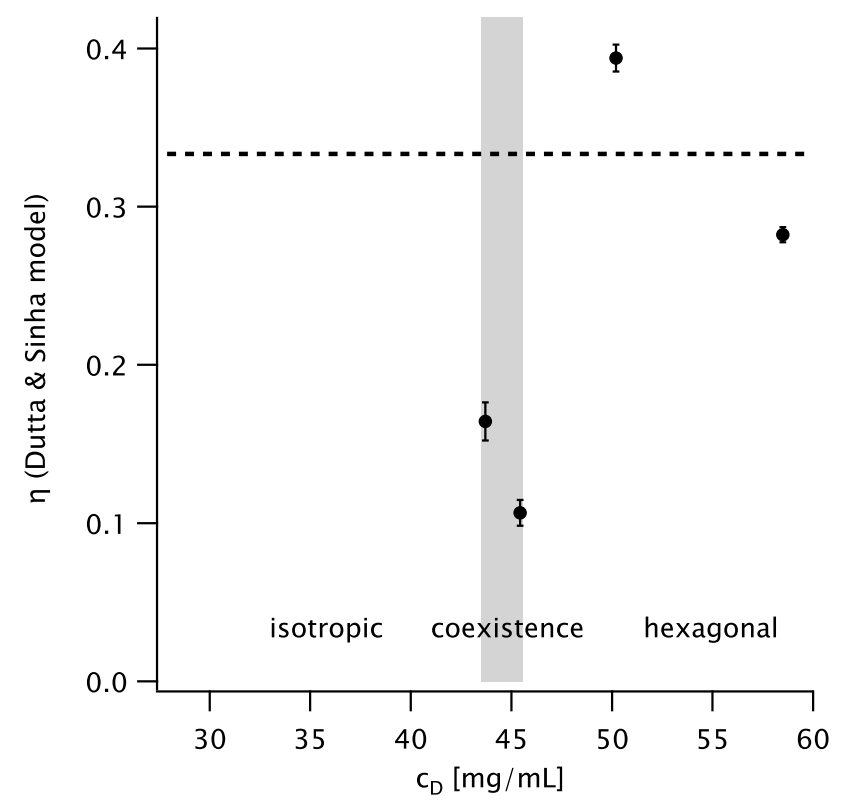

Figure 5: Fluctuation parameter $\eta$ in the D \& $\mathrm{S}$ model as a function of the Dextran concentration $c_{D}$.

The values of $\eta$ extracted from the $\mathrm{D} \& \mathrm{~S}$ model are shown in Figure 5. In the hexagonal phase, they are 0.28 and 0.39 , so we can estimate an average value $\eta_{\text {avg }}=1 / 3$, which happens to be the maximum value that $\eta$ can reach in the KTHNY scenario. Plugging it into (2) and setting the final factor to 1 (under the assumption that $\lambda \gg \mu$, to be justified below) yields a shear modulus:

$$
\mu \simeq 4 \pi \frac{k_{B} T}{d^{2}}=0.09 \frac{k_{B} T}{\mathrm{~nm}^{2}}=3.610^{-4} \mathrm{Pam}
$$

From the osmotic pressure dependence. ${ }^{29}$ we estimate the bulk modulus at

$$
\mu+\lambda=6.510^{-3} \mathrm{Pam}
$$

about 18 times higher than the shear modulus. For context, this ratio is 3 in hexagonal lyotropic liquid crystals, ${ }^{37} 10$ in magnetic colloids at the air-water interface ${ }^{38}$ and about 12 in decanol monolayers. ${ }^{39}$

The values of $\eta$ estimated in the coexistence regime are unreliable, since the wide tails associated to high $\eta$ are difficult to distinguish from the "bump" of the isotropic phase. The coexistence model thus yields $\eta \sim 0.1-0.15$, as shown in Figure 5. The pure D \& $\mathrm{S}$ model, on the other hand, gives $\eta \sim 0.5-0.6$ (data not plotted.)

In the isotropic phase, the structure factor is well described by a hard core interaction, in agreement with recent macroscopic results. ${ }^{40}$ The osmotic pressure is much lower than expected from the concentration of Dextran, so the latter is probably not completely excluded from the membranes, as it happens in bulk sys- 
tems. $^{41}$

Self-assembled membranes of stiff $f d$ viruses represent an experimental quasi-twodimensional system of isotropic objects that does not follow the KTHNY scenario. In particular, they exhibit a direct transition between the crystalline and the fluid phases. We see no evidence for a hexatic phase, in contrast with literature results for bulk phases of a semi-flexible $f d$ viruses $^{25}$ and for dense DNA phases. ${ }^{15-17}$

This result is puzzling, in view of the similarity between our $f d$ viruses and the hexatic systems cited above. A notable difference is that the former is stiffer than dsDNA and also than the wild-type $f d$ virus strains used in bulk studies. Our findings highlight the importance of flexibility in stabilizing the hexatic phase of elongated objects. This is a new result, since to our knowledge the role of flexibility has never been invoked in the literature in this context.

Acknowledgement The SOLEIL synchrotron is acknowledged for the provision of beamtime (experiment 20150494) and Javier Perez for his support. This work was supported by the ANR under contract MEMINT (2012-BS04-0023).

\section{Supporting Information Avail- able}

The following files are available free of charge.

- SI_Constantin_2018.pdf: details on the fitting procedure, the evaluation of the form factor and of the osmotic pressure, the comparison of data from dilute and concentrated suspensions and on the implementation of the crystal model.

\section{References}

(1) von Grünberg, H. H.; Keim, P.; Maret, G. In Soft Matter; Gompper, G., Schick, M., Eds.; Wiley-VCH Verlag GmbH \& Co. KGaA, 2007; Vol. 3; Chapter 2, pp 41-86.
(2) Heiney, P. A.; Stephens, P. W.; Birgeneau, R. J.; Horn, P. M.; Moncton, D. E. X-ray scattering study of the structure and freezing transition of monolayer xenon on graphite. Physical Review B 1983, 28, 6416-6434.

(3) Stephens, P. W.; Heiney, P. A.; Birgeneau, R. J.; Horn, P. M.; Moncton, D. E.; Brown, G. S. High-resolution xray-scattering study of the commensurateincommensurate transition of monolayer Kr on graphite. Physical Review B 1984, 29, 3512-3532.

(4) Dimon, P.; Horn, P. M.; Sutton, M.; Birgeneau, R. J.; Moncton, D. E. Firstorder and continuous melting in a twodimensional system: Monolayer xenon on graphite. Physical Review B 1985, 31, 437-447.

(5) Keim, P.; Maret, G.; Herz, U.; von Grünberg, H. H. Harmonic Lattice Behavior of Two-Dimensional Colloidal Crystals. Physical Review Letters 2004, 92, 215504.

(6) von Grünberg, H. H.; Keim, P.; Zahn, K.; Maret, G. Elastic behavior of a twodimensional crystal near melting. Physical Review Letters 2004, 93, 255703.

(7) Brodin, A.; Nych, A.; Ognysta, U.; Lev, B.; Nazarenko, V.; Skarabot, M.; Musevic, I. Melting of 2D liquid crystal colloidal structure. Condensed Matter Physics 2010, 13, 33601.

(8) Thorneywork, A. L.; Abbott, J. L.; Aarts, D. G. A. L.; Dullens, R. P. A. TwoDimensional Melting of Colloidal Hard Spheres. Physical Review Letters 2017, 118, 158001.

(9) Koltover, I.; Raedler, J. O.; Salditt, T.; Rothschild, K. J.; Safinya, C. R. Phase behavior and interactions of the membraneprotein bacteriorhodopsin. Physical Review Letters 1999, 82, 3184-3187. 
(10) Constantin, D. Membrane-mediated repulsion between gramicidin pores. Biochimica et Biophysica Acta (BBA) Biomembranes 2009, 1788, 1782-1789.

(11) Constantin, D.; Pansu, B.; Impéror, M.; Davidson, P.; Ribot, F. Repulsion Between Inorganic Particles Inserted Within Surfactant Bilayers. Phys. Rev. Lett. 2008, 101, 098101.

(12) Pansu, B.; Lecchi, A.; Constantin, D.; Impéror-Clerc, M.; Veber, M.; Dozov, I. Insertion of Gold Nanoparticles in Fluid Mesophases: Size Filtering and Control of Interactions. The Journal of Physical Chemistry $C$ 2011, 115, 17682-17687.

(13) Petukhov, A. V.; van der Beek, D.; Dullens, R. P. A.; Dolbnya, I. P.; Vroege, G. J.; Lekkerkerker, H. N. W. Observation of a hexatic columnar liquid crystal of polydisperse colloidal disks. Physical Review Letters 2005, 95, 077801.

(14) Pal, A.; Kamal, M. A.; Raghunathan, V. A. Observation of the Chiral and Achiral Hexatic Phases of Selfassembled Micellar polymers. Scientific Reports 2016, 6, 32313.

(15) Podgornik, R.; Strey, H. H.; Gawrisch, K.; Rau, D. C.; Rupprecht, A.; Parsegian, V. A. Bond orientational order, molecular motion, and free energy of high-density DNA mesophases. Proceedings of the National Academy of Sciences 1996, 93, 4261-4266.

(16) Strey, H. H.; Wang, J.; Podgornik, R.; Rupprecht, A.; Yu, L.; Parsegian, V. A.; Sirota, E. B. Refusing to Twist: Demonstration of a Line Hexatic Phase in DNA Liquid Crystals. Physical Review Letters 2000, 84, 3105-3108.

(17) Yasar, S.; Podgornik, R.; Valle-Orero, J.; Johnson, M. R.; Parsegian, V. A. Continuity of states between the cholesteric $\rightarrow$ line hexatic transition and the condensation transition in DNA solutions. Scientific Reports 2014, 4, 6877.
(18) Leforestier, A.; Livolant, F. The Bacteriophage Genome Undergoes a Succession of Intracapsid Phase Transitions upon DNA Ejection. Journal of Molecular Biology 2010, 396, 384-395.

(19) Modlińska, A.; Alsayed, A. M.; Gibaud, T. Condensation and dissolution of nematic droplets in dispersions of colloidal rods with thermo-sensitive depletants. Scientific reports 2015, 5, 18432.

(20) Barry, E.; Dogic, Z. Entropy driven self-assembly of nonamphiphilic colloidal membranes. Proceedings of the National Academy of Sciences 2010, 107, 10348 10353.

(21) Kang, L.; Gibaud, T.; Dogic, Z.; Lubensky, T. C. Entropic forces stabilize diverse emergent structures in colloidal membranes. Soft Matter 2015, 12, 386-401.

(22) (a) Gibaud, T.; Barry, E.; Zakhary, M. J.; Henglin, M.; Ward, A.; Yang, Y.; Berciu, C.; Oldenbourg, R.; Hagan, M. F.; Nicastro, D.; Meyer, R. B.; Dogic, Z. Reconfigurable self-assembly through chiral control of interfacial tension. Nature 2012, 481, 348-351; (b) Gibaud, T. Filamentous phages as building blocks for reconfigurable and hierarchical selfassembly. Journal of Physics: Condensed Matter 2017, 29, 493003.

(23) Sharma, P.; Ward, A.; Gibaud, T.; Hagan, M. F.; Dogic, Z. Hierarchical organization of chiral rafts in colloidal membranes. Nature 2014, 513, 77-80.

(24) Purdy, K.; Dogic, Z.; Fraden, S.; Rühm, A.; Lurio, L.; Mochrie, S. Measuring the nematic order of suspensions of colloidal fd virus by x-ray diffraction and optical birefringence. Physical Review E 2003, 67, 031708.

(25) Grelet, E. Hexagonal Order in Crystalline and Columnar Phases of Hard Rods. Physical Review Letters 2008, 100, 168301. 
(26) Barry, E.; Beller, D.; Dogic, Z. A model liquid crystalline system based on rodlike viruses with variable chirality and persistence length. Soft Matter 2009, 5, 25632570 .

(27) Sambrook, J.; Russell, D. W. Molecular cloning: a laboratory manual 3rd edition. Coldspring-Harbour Laboratory Press, UK 2001,

(28) Asakura, S.; Oosawa, F. Interaction between particles suspended in solutions of macromolecules. Journal of polymer science 1958, 33, 183-192.

(29) See the Supporting Information for fitting details, the evaluation of the form factor and osmotic pressure, the comparison of data from dilute and concentrated suspensions and for the implementation of the crystal model.

(30) Rosenfeld, Y. Free-energy model for the inhomogeneous hard-sphere fluid in D dimensions: Structure factors for the harddisk $(\mathrm{D}=2)$ mixtures in simple explicit form. Physical Review A 1990, 42, 59785989 .

(31) Ref. 30, Eq. (6.8).

(32) Dutta, P.; Sinha, S. K. Analytic form for the static structure factor for a finite twodimensional harmonic lattice. Physical Review Letters 1981, 47, 50-53.

(33) Ref. 32, Eq. (5).

(34) Ref. 2, Eq. (9).

(35) Livney, Y. D.; Ramon, O.; Kesselman, E.; Cogan, U.; Mizrahi, S.; Cohen, Y. Swelling of dextran gel and osmotic pressure of soluble dextran in the presence of salts. Journal of Polymer Science Part B: Polymer Physics 2001, 39, 2740-2750.

(36) Nelson, D. R.; Halperin, B. I. Dislocationmediated melting in two dimensions. Physical Review B 1979, 19, 2457-2484.
(37) Impéror-Clerc, M.; Davidson, P. An X-ray scattering study of flow-aligned samples of a lyotropic liquid-crystalline hexagonal phase. The European Physical Journal BCondensed Matter and Complex Systems 1999, 9, 93-104.

(38) Zahn, K.; Wille, A.; Maret, G.; Sengupta, S.; Nielaba, P. Elastic Properties of 2D Colloidal Crystals from Video Microscopy. Phys. Rev. Lett. 2003, 90, 155506 .

(39) Zakri, C.; Renault, A.; Rieu, J.-P.; Vallade, M.; Berge, B.; Legrand, J.-F.; Vignault, G.; Grübel, G. Determination of the in-plane elastic tensor of crystalline decanol monolayers on water by x-ray diffraction. Phys. Rev. B 1997, 55, 1416314172 .

(40) Grelet, E. Hard-Rod Behavior in Dense Mesophases of Semiflexible and Rigid Charged Viruses. Physical Review X 2014, 4, 021053 .

(41) Dogic, Z.; Purdy, K.; Grelet, E.; Adams, M.; Fraden, S. Isotropic-nematic phase transition in suspensions of filamentous virus and the neutral polymer Dextran. Physical Review E 2004, 69, 051702. 